\title{
Scheme of Optical Fiber Temperature Sensor Employing Deep-Grooved Process Optimization
}

\author{
Yu LIU, Cong LIU*, Gaolin XIANG, Ruijie WANG, Yibing WANG, \\ Lei Xiang, Linzhi WU, and Song LIU \\ Chongqing Municipal Level Key Laboratory of Photoelectronic Information Sensing \& Transmitting Technology, \\ Chongqing University of Posts and Telecommunications, Chongqing, 400065, China \\ *Corresponding author: Cong LIU_Ｅmail: sequential1990@sina.com
}

\begin{abstract}
To optimize the optical fiber temperature sensor employing the deep-grooved process, a novel scheme was proposed. Fabricated by the promising $\mathrm{CO}_{2}$ laser irradiation system based on the two-dimensional scanning motorized stage with high precision, the novel deep-grooved optical fiber temperature sensor was obtained with its temperature sensitivity of the transmission attenuation $-0.107 \mathrm{~dB} /{ }^{\circ} \mathrm{C}$, which was 18.086 times higher than the optical fiber sensor with the normal depth of grooves while other parameters remained unchanged. The principal research and experimental testing showed that the designed temperature sensor measurement unit had the ability of high sensitivity in transmission attenuation and insensitivity to the wavelength, which offers possible applications in engineering.
\end{abstract}

Keywords: OFTS, temperature sensitivity, transmission attenuation, deep-grooved process

Citation: Yu LIU, Cong LIU, Gaolin XIANG, Ruijie WANG, Yibing WANG, Lei Xiang, et al., "Scheme of Optical Fiber Temperature Sensor Employing Deep-Grooved Process Optimization," Photonic Sensors, 2015, 5(1): 6-11.

\section{Introduction}

The temperature sensor fabricated by the optical fiber materials has attracted significant attentions of researchers, owing to the advantages of their light volume, high sensitivity, short response time, anti-electromagnetic interference, and corrosion resistance in recent years [1]. Typical fiber temperature sensor devices such as fiber Bragg gratings (FBGs) [2], long period fiber gratings (LPFGs) [3], optical wireless sensing sensors [4], and photonic crystal fibers (PCFs) [5] have been developed with various fiber optic refractive index (RI) sensors. Various types of temperature sensors in the optical fiber have been employed to achieve superior precision, which still have intrinsic defects such as the poor stability, numerous cycle numbers, long length, and low sensitivity to the temperature [2-5]. The single mode fiber (SMF) is demonstrated as one classical material which is convenient for fabrication with very low costs at the same precision level [6]. Therefore, the process optimization of the SMF temperature sensor according to intensity modulation possesses a certain value in practical sensing applications.

In our work, a novel optical fiber temperature sensor (OFTS) employing the deep-grooved process which was fabricated by the $\mathrm{CO}_{2}$ laser irradiation system to carve the grooves with certain depth was proposed. This deep-grooved OFTS obtained a large dip attenuation of $-33.85 \mathrm{~dB}$ at the resonant wavelength of $1426.7 \mathrm{~nm}$, which was achieved in a

Received: 5 November 2014 / Revised version: 8 December 2014

C The Author(s) 2014. This article is published with open access at Springerlink.com

DOI: $10.1007 / \mathrm{s} 13320-014-0229-5$

Article type: Regular 
standard SMF. Based on realizing the similar sensitivity of the resonant wavelength, the sensitivity of transmission attenuation could be increased by deepening the depth of the grooves on one side of the fiber when the temperature increased.

\section{Experimental setup and principle}

Our experimental platform for the OFTS fabrication is shown in Fig. 1. The $\mathrm{CO}_{2}$ laser irradiation system consisted of a $\mathrm{CO}_{2}$ laser (SYNRAD 48-1) along the $z$ axis with a maximum output power of $10 \mathrm{~W}$, an electric shutter for switching the laser beam, an infrared lens, a four-times beam expander, and a two-dimensional high-precision motorized stage (Newport VP-25X). The standard SMF (Corning SMF-28) was situated in the focal plane of the $\mathrm{CO}_{2}$ laser beam which was precisely controlled by the two-dimensional stage. An amplified spontaneous emission (ASE) broad-band light source and an optical spectrum analyzer (YOKOGAWA AQ6370C) were used to monitor the transmission spectrum of the OFTS during the grating fabrication. The left end of the fiber was fixed by a fiber holder, and a small weight was attached to the right free end for providing the constant tensile strain.

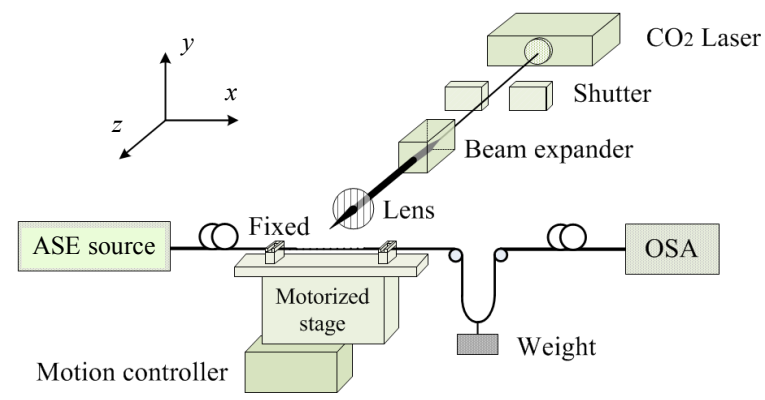

Fig. 1 Schematic diagram of the experimental setup for OFTS fabrication.

Controlled by an intelligent program, the motorized stage horizontally moved by a grating period along the fiber axis ( $x$ axis). The OFTS was carried out for realizing $N$ times ( $N$ is the grating period number) of this scanning and shifting process. Until the OFTS had been produced in a high quality, the process mentioned above was repeated for a certain number of cycles ( $M$ times). In our experiments, the measured diameter of the focused $\mathrm{CO}_{2}$ laser beam spot was only about $30 \mu \mathrm{m}$, the motorized stage was moved with a speed of $0.5 \mathrm{~mm} / \mathrm{s}$ for scanning on the fiber along the $Y$ axis, and the average output power of the $\mathrm{CO}_{2}$ laser was $0.5 \mathrm{~W}$.

The refractive index modulation of the stretched OFTS carved by the $\mathrm{CO}_{2}$ laser beam has two components and can be expressed as follows:

$$
\Delta n=\Delta n_{\text {strain }}+\Delta n_{\text {temperature }}
$$

where $\Delta n_{\text {strain }}$ is the refractive index perturbation, due to the photoelastic effect, induced by the difference between the grooved and ungrooved regions [7]; $\Delta n_{\text {temperature }}$ is an additional refractive index perturbation induced by the thermal strain in the asymmetric OFTS, which can increase with an increase in the temperature and can be expressed as follows:

$$
\Delta n_{\text {temperature }}=\Delta n_{\text {groove }}+\Delta n_{\text {microbend }}+\Delta n_{\text {residual }}
$$

where $\Delta n_{\text {grove }}$ is the original refractive index perturbation induced by the periodic grooves; $\Delta n_{\text {microbend }}$ is the refractive index perturbation induced by the microbends produced from the stretched OFTS carved by the $\mathrm{CO}_{2}$ laser beam for one-side grooves [8]; $\Delta n_{\text {residual }}$ is the original refractive index perturbation induced by the residual stress due to the local high temperature [9].

As shown in Fig. 2, when the temperature increases, the deformation of grooves generated along the axial direction, which gave rise to the modulation of in-fiber refractive index. From the schematic diagram, it is obvious that the angle of the side wall in the groove decreased with an increase in the depth of the groove, resulting in the larger deformation of the groove. From Fig. 2(b), the mathematic relation can be expressed as follows:

$$
\begin{gathered}
\frac{1}{2} W=\frac{1}{2}(2 \theta) \cdot\left(R_{0}-D\right)=\theta \cdot\left(R_{0}-D\right) \\
\sin \theta=\frac{W_{A}}{R_{0}}
\end{gathered}
$$


where $W$ is the width of the groove, $\theta$ is the angle of the side wall in the groove, which ranges from zero to $\pi / 2, R_{0}$ is the radius of the curving groove, and $W_{A}$ is the fixed width from the ungrooved section to the center of the grooved section, which figures out that the angle $\theta$ of the side wall in the groove decreases with an increase in $R_{0}$. Substituting (4) into (3), we obtain (5) as follows:

$$
D \approx \frac{W_{A}}{\sin \theta}-\frac{W}{2 \theta}
$$

which apparently points out that the angle $\theta$ of the side wall in the groove decreases with an increase in $D$, where $\Delta n_{\text {groove }}$ can be increased by the deformation of grooves. When $\theta$ keeps very close to zero, the deformation approaches to the minimum value, for counteracting in part by the interference produced by the thermal expansion in the temperature measurement [10].

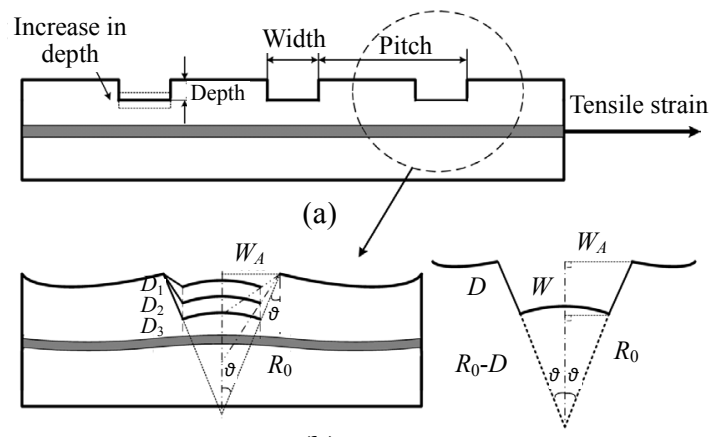

(b)

Fig. 2 Schematic of the $\mathrm{CO}_{2}$-laser-carved OFTS: (a) before and (b) after the temperature measurement, where the angle of the side wall in the groove decreases with an increase in the depth of the groove.

\section{Results and discussion}

We demonstrated a novel deep grooved OFTS $\left(\mathrm{OFTS}_{1}\right)$, as shown in Fig. 3. With a low insertion loss of about $3 \mathrm{~dB}$, one attenuation dip (the resonant wavelength and the peak transmission attenuation of dip were $\lambda_{1}=1426.7 \mathrm{~nm}$ and $A_{1}=-33.85 \mathrm{~dB}$ ), and this novel deep grooved OFTS with $d=15 \mu \mathrm{m}$, was produced enough depth on one side of the optical fiber. As shown in Fig. 3, the periodic grooves with a grating pitch of $420 \mu \mathrm{m}$ were carved on the fiber.
Owing to the photoelastic effect, the grooves could induce periodic refractive index modulations along the fiber axis, which were made into an OFTS. When the depth was increased more than $15 \mu \mathrm{m}$ within our platform, the sample might molder immediately as the transmission spectrum disappeared.

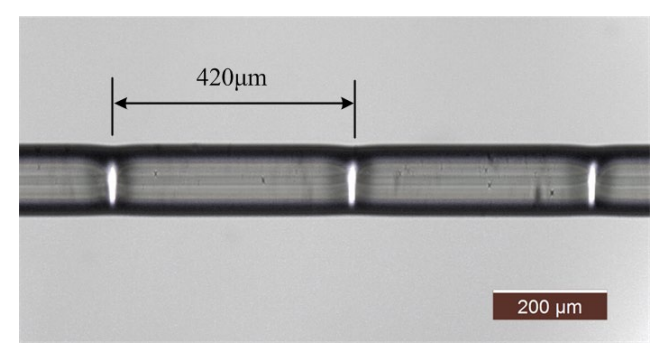

Fig. 3 Charge-coupled-device (CCD) photograph of the OFTS with periodic grooves with a grating pitch of $420 \mu \mathrm{m}$.

Resulting from the abrupt material deformation, the grooved region of the OFTS is a stress concentration area [11]. Accordingly, the coupling from the fundamental core mode to the cladding mode is enhanced with an increase in the temperature, which will observably change the transmission attenuation of the OFTS, as shown in Fig. 4.

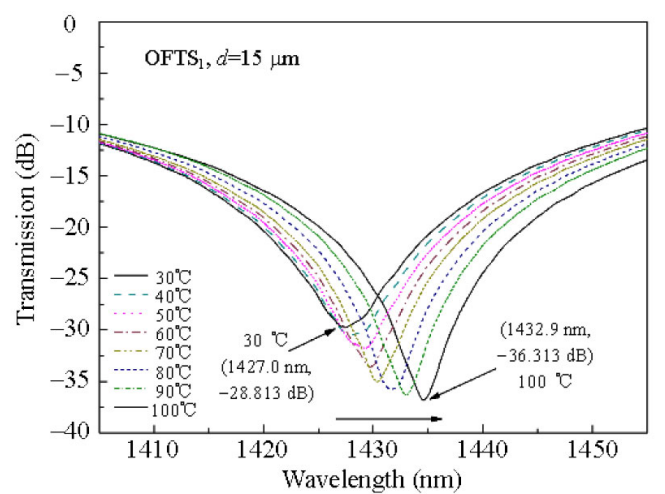

Fig. 4 Transmission spectrum evolutions of the OFTS with the temperature.

To clarify the difference between the temperature characteristics of the OFTSs with different depths of the periodic grooves, another two OFTSs $\left(\mathrm{OFTS}_{2}\right.$ and $\left.\mathrm{OFTS}_{3}\right)$ with less deep grooves were produced in the same type of the standard single mode fiber (YOF Inc.) $(d=10 \mu \mathrm{m}$ and $5 \mu \mathrm{m}$, respectively). With a low insertion loss of about $2 \mathrm{~dB}$, 
the attenuation dips (the resonant wavelength and the peak transmission attenuation of $\mathrm{Dip}_{2}$ and $\mathrm{Dip}_{3}$ were $\lambda_{2}=1502.00 \mathrm{~nm}, A_{2}=-36.82 \mathrm{~dB}, \lambda_{3}=$ $1588.9 \mathrm{~nm}$, and $A_{3}=-32.78 \mathrm{~dB}$, respectively) were observed from the transmission spectrum of $\mathrm{OFTS}_{2}$ and $\mathrm{OFTS}_{3}$. All of the $\mathrm{OFTS}_{1}, \mathrm{OFTS}_{2}$, and OFTS had the same grating pitch and grating period, which were $420 \mu \mathrm{m}$, and $N=30$.

Figure 5(a) shows the results obtained from a number of temperature sensing tests employing wavelength and transmission features of the $\mathrm{OFTS}_{1}$, $\mathrm{OFTS}_{2}$, and $\mathrm{OFTS}_{3}$. The resonant wavelength of all the three OFTSs shifts were linearly towards the long wavelength. In Fig. 5(a), Curve 1 shows the measured response of period $\mathrm{OFTS}_{1}$ over the temperature range from $30{ }^{\circ} \mathrm{C}$ to $100{ }^{\circ} \mathrm{C}$. With an

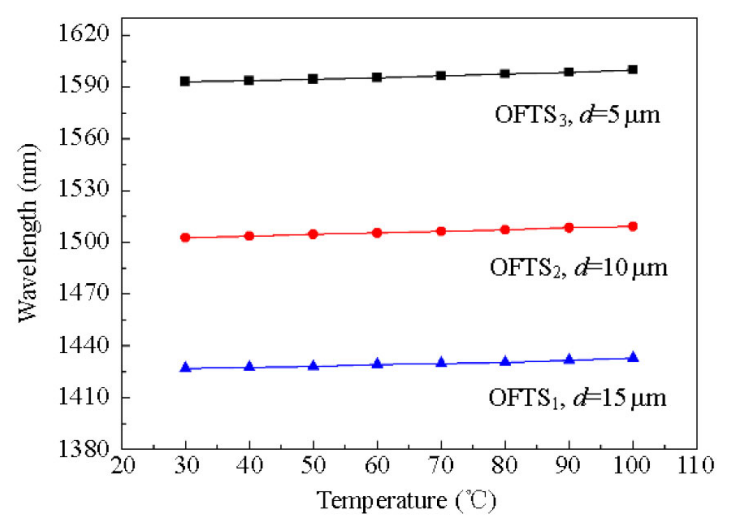

(a)

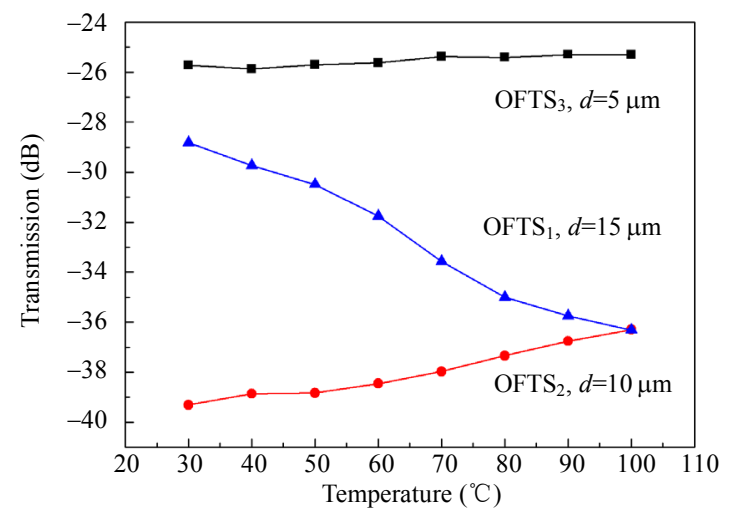

(b)

Fig. 5 Relationships of the resonant wavelength and peak transmission attenuation with the temperature: (a) resonant wavelength and (b) peak transmission attenuation as functions of the temperature for $\mathrm{OFTS}_{1}, \mathrm{OFTS}_{2}$, and $\mathrm{OFTS}_{3}$, respectively. increase in the temperature, the resonant wavelength reached the red shift about $+5.9 \mathrm{~nm}$. Utilizing the wavelength shift as the basis of the temperature measurement, the $\mathrm{OFTS}_{1}$ exhibited a sensitivity as large as $84.3 \mathrm{pm} /{ }^{\circ} \mathrm{C}$ in the range of $30{ }^{\circ} \mathrm{C}-100{ }^{\circ} \mathrm{C}$. Similarly, the temperature sensitivity of the resonant wavelengths, as shown in Curves 2 and 3, for the samples $\mathrm{OFTS}_{2}$ and $\mathrm{OFTS}_{3}$ were $93.6 \mathrm{pm} /{ }^{\circ} \mathrm{C}$ and $97.7 \mathrm{pm} /{ }^{\circ} \mathrm{C}$, respectively.

Apart from the wavelength shift occurring at different temperatures, the peak transmission attenuation of OFTS $_{1}$ changed from $-28.83 \mathrm{~dB}$ to $-36.31 \mathrm{~dB}$, by which the temperature sensitivity of the transmission attenuation $-0.107 \mathrm{~dB} /{ }^{\circ} \mathrm{C}$ was obtained, as shown in Fig.5(b), Curve 1. Similarly, as shown in Fig. 5(b), the temperature sensitivities of the transmission attenuation, as shown in Curves 2 and 3 , for the samples $\mathrm{OFTS}_{2}$ and $\mathrm{OFTS}_{3}$ were $0.043 \mathrm{~dB} /{ }^{\circ} \mathrm{C}$ and $0.00607 \mathrm{~dB} /{ }^{\circ} \mathrm{C}$, respectively.

Temperature characteristics of the three OFTSs under different depths of grooves are summarized in Fig. 6. As shown in Fig. 6, with an increase in the depth of grooves (scanning cycles), the temperature sensitivities of the resonant wavelengths from all three OFTSs were very close to each other. On the contrary, the lower curve in Fig. 6 illustrates the absolute sensitivity value of transmission attenuation of all three OFTSs vary widely, in which $\mathrm{OFTS}_{2}$ was 7.1 times higher than $\mathrm{OFTS}_{3}$, and $\mathrm{OFTS}_{1}$ was 18.09 times higher than OFTS $_{3}$. Consequently, it indicates that the temperature sensitivity of transmission attenuation can be increased by creating deeper grooves during the fabrication.

The research by Vaziri et al. [12] showed a good linearity for the coefficient of strain increased with an increase in the depth of grooves. Experiments in this paper showed the dissimilar conclusion that the absolute value of sensitivity may comply with the linearity for the depth of grooves, not the original sensitivity. Possible reasons for the differences could 
be theoretical derivation or manufacturing operation. The functional relationship between the depth of grooves and temperature sensitivity maybe more complicated than that of the existing researches, which deserves further studies.

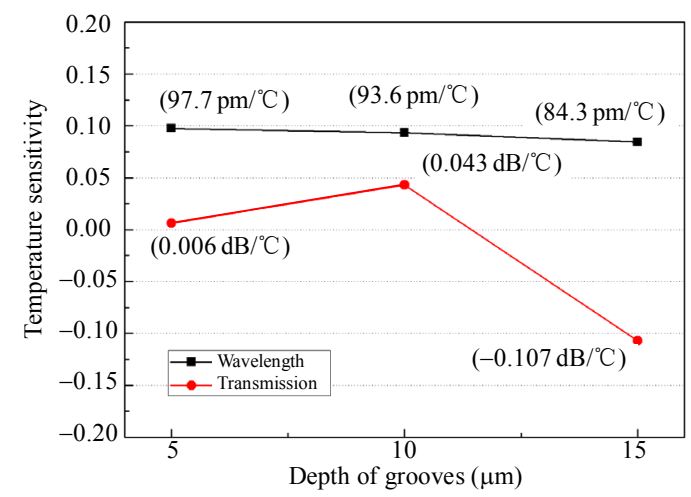

Fig. 6 Temperature sensitivity versus different depths of grooves for the resonant wavelength and peak transmission attenuation of three OFTSs.

\section{Conclusions}

The novel deep-grooved optical fiber temperature sensor indicates unique temperature sensing characteristics. Based on realizing the similar sensitivity of the resonant wavelength, the sensitivity of transmission attenuation could be increased by deepening the depth of the grooves on one side of the fiber when temperature increased. Moreover, the sensitivity of the novel OFTS with $d$ $=15 \mu \mathrm{m}$ was 18.09 times higher than that of the congeneric OFTS with $d=5 \mu \mathrm{m}$. Consequently, such an OFTS can be utilized as a temperature sensor based on the intensity modulation, which will be one of the simple and alternative process optimizations based on the intensity modulation in the engineering application.

\section{Acknowledgment}

This work was supported in part by the National Natural Science Foundation of China under Grant 51175535, in part by the Natural Science Foundation Project of CQ CSTC under Grant CSTC2012jjB40009, and in part by the Key Laboratory of Optoelectronic Devices and Systems of Ministry of Education and Guangdong
Province.

Open Access This article is distributed under the terms of the Creative Commons Attribution License which permits any use, distribution, and reproduction in any medium, provided the original author(s) and source are credited.

\section{References}

[1] X. Fang, C. Liao, and D. Wang, "Femtosecond laser fabricated fiber Bragg grating in microfiber for refractive index sensing," Optics Letters, 2010, 35(7): 1007-1009.

[2] B. Zhang and M. Kahrizi, "High-temperature resistance fiber Bragg grating temperature sensor fabrication," IEEE Sensors Journal, 2007, 7(4): 586-591.

[3] Y. Wang, W. Jin, and D. Wang, "Unique temperature sensing characteristics of $\mathrm{CO}_{2}$-laser-notched long-period fiber gratings," Optics and Lasers in Engineering, 2009, 47(10): 1044-1048.

[4] W. Gan and Y. Wang, "Study of isolated contact temperature monitoring system based on optical wireless sensing technology," Photonic Sensors, 2014, 4(2): 108-112.

[5] X. Chen, Y. Yu, X. Xu, Q. Huang, Z. Ou, J. Wang, et al., "Temperature insensitive bending sensor based on in-line Mach-Zehnder interferometer," Photonic Sensors, 2014, 4(3): 193-197.

[6] J. Zhu, A. P. Zhang, T. Xia, and S. He, "Fiber-optic high-temperature sensor based on thin-core fiber modal interferometer," IEEE Sensors Journal, 2010, 10(9): 1415-1418.

[7] C. Lin, L. Wang, and G. Chern, "Corrugated long-period fiber gratings as strain, torsion, and bending Sensors," Journal of Lightwave Technology, 2001, 19(8): 1159-1168.

[8] C. Lin, Q. Li, A. A. Au, Y. Jiang, E. Wu, and H. Lee, "Strain-induced thermally tuned long-period fiber gratings fabricated on a periodically corrugated," Journal of Lightwave Technology, 2004, 22(7): 1818-1827.

[9] Y. Wang, D. N. Wang, W. Jin, Y. Rao, and G. Peng, "Asymmetric long period fiber gratings fabricated by use of $\mathrm{CO}_{2}$ laser to carve periodic grooves on the optical fiber," Applied Physics Letters, 2006, 89(15): 151105-1-151105-3.

[10] Y. Liu, L. Zeng, Y. Lu, S. Liu, and Z. Huang, "Intensity-modulated bending sensors based on rare- 
earth-doped fibers," Acta Physica Sinica, 2011, 60(10): 372-376.

[11] Y. Yu, X. Li, X. Hong, Y. Deng, K. Song, Y. Geng, et al., "Some features of photonic crystal fiber temperature sensor based on liquid ethanol filling," Optics Express, 2010, 18(15): 15383-15388.

[12] M. Vaziri and C. Chen, "Optical-fiber strain sensors with asymmetric etched structures," Applied Optics, 1993, 32(31): 6399-6406. 